\title{
Search for Low Mass Dark Matter Particles with the CRESST Experiment
}

Cenk Türkoğlu ${ }^{* 4,5} ;$ G. Angloher ${ }^{1}$, P. Bauer ${ }^{1}$, A. Bento ${ }^{1,8}$, C. Bucci ${ }^{2}$, L. Canonica ${ }^{2,9}$, X. Defay ${ }^{3}$, A. Erb ${ }^{3,10}$, F. v. Feilitzsch ${ }^{3}$, N. Ferreiro lachellini ${ }^{1}$, P. Gorla ${ }^{2}$, A. Gütlein ${ }^{4,5}$, D. Hauff ${ }^{1}$, J. Jochum ${ }^{6}$, M. Kiefer ${ }^{1}$, H. Kluck ${ }^{4,5}$, H. Kraus ${ }^{7}$, J.-C. Lanfranchi ${ }^{3}$,

A. Langenkämper ${ }^{3}$, J. Loebell ${ }^{6}$, M. Mancuso $^{1}$, E. Mondragon ${ }^{3}$, A. Münster ${ }^{3}$, C. Pagliarone ${ }^{2}$, F. Petricca ${ }^{1}$, W. Potzel ${ }^{3}$, F. Pröbst ${ }^{1}$, R. Puig ${ }^{4,5}$, F. Reindl ${ }^{4,5}$, J. Rothe ${ }^{1}$, K. Schäffner ${ }^{2,11}$, J. Schieck ${ }^{4,5}$, S. Schönert ${ }^{3}$, W. Seidel ${ }^{1} ;$ M. Stahlberg ${ }^{4,5}$, L. Stodolsky ${ }^{1}$, C. Strandhagen ${ }^{6}$, R. Strauss ${ }^{1}$, A. Tanzke ${ }^{1}$, H.H. Trinh Thi ${ }^{3}$, M. Uffinger ${ }^{6}$, A. Ulrich ${ }^{3}$, I. Usherov ${ }^{6}$, S. Wawoczny ${ }^{3}$, M. Willers ${ }^{3}$, M. Wüstrich ${ }^{1}$

1 Max-Planck-Institut für Physik, D-80805 München, Germany

2 INFN, Laboratori Nazionali del Gran Sasso, I-67010 Assergi, Italy

3 Physik-Department and Excellence Cluster Universe, Technische Universität München, D-85747 Garching, Germany

4 Institut für Hochenergiephysik der Österreichischen Akademie der Wissenschaften, A-1050 Wien, Austria

5 Atominstitut, Vienna University of Technology, A-1020 Wien, Austria

6 Eberhard-Karls-Universität Tübingen, D-72076 Tübingen, Germany

7 Department of Physics, University of Oxford, Oxford OX1 3RH, United Kingdom

8 Also at: LIBPhys, Departamento de Fisica, Universidade de Coimbra, P3004 516 Coimbra,

Portugal

9 Also at: Massachusetts Institute of Technology, Cambridge, MA 02139, USA

10 Also at: Walther-Meißner-Institut für Tieftemperaturforschung, D-85748 Garching, Germany

11 Also at: GSSI-Gran Sasso Science Institute, 67100, L’Aquila, Italy

It has been proven by several astronomical observations that dark matter exists, but no particle candidates have been observed yet. The CRESST experiment aims to directly detect dark matter particles elastically scattering off nuclei in $\mathrm{CaWO}_{4}$ crystals which are operated at $\mathrm{mK}$ temperatures. With nuclear recoil energy thresholds as low as $0.3 \mathrm{keV}$ [2] and $0.6 \mathrm{keV} \mathrm{[3],} \mathrm{for} \mathrm{the} \mathrm{detector}$ modules LISE and TUM40, respectively, CRESST is ideally suited for the detection of low-mass dark matter particles [5]. Additionally, the radiopurity of the crystals is another important factor for the detector performance. For a detailed understanding of the detector backgrounds, we simulate the radioactive contaminations of the TUM40 detector module with Geant 4 . The outcome of this simulation will be vital for the CRESST-III experiment. In this contribution, we discuss our results of the search for dark matter and dark photons achieved with the detector module Lise of CRESST-II. We will discuss the status of CRESST-III Phase 1 which started taking data in 2016.

The European Physical Society Conference on High Energy Physics

5-12 July

Venice, Italy 


\section{Dark Matter and Direct Detection}

One of the biggest mysteries of our universe is the nature of dark matter. Different observations prove that dark matter exists. Over the years, many theory models have been constructed to explain dark matter. Particle candidates with different masses, crosssections, production, annihilation and scattering mechanisms have been proposed [1]. Nucleon dark matter cross-sections for some dark matter candidates are shown in Figure 1. Direct detection experiments search for dark matter scattering off nuclei of the target material. The search technique changes depending on the sensitivity of the mass range the physicists are interested in. In Figure 2 , most current results of the dark matter particle nucleon cross sections from different experiments are shown with respect to the dark matter particle mass. For higher dark matter particle masses, exposure is an important limiting factor whereas for lighter dark matter particle masses, de-

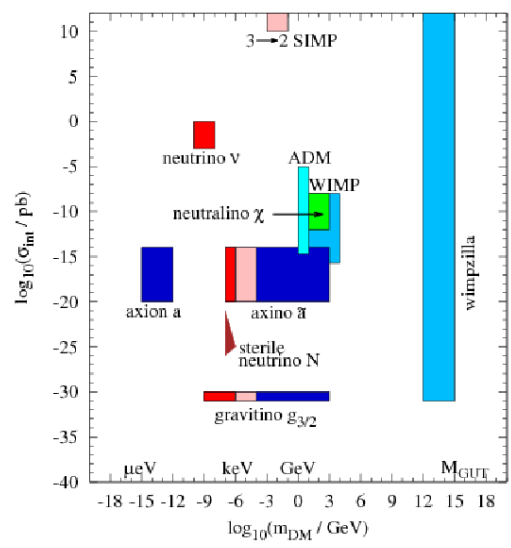

Figure 1: Nucleon to dark matter particle cross-sections of different dark matter theory models drawn with respect to the predicted dark matter masses [1]. tection threshold is more crucial. One of the experiments which focuses on the low mass dark matter particle search is the CRESST experiment.

\section{The CRESST-II Experiment}

CRESST stands for "Cryogenic Rare Event Search with Superconducting Thermometers". The experiment is located at Laboratori Nazionali del Gran Sasso (LNGS) in Italy. CRESST is a direct detection experiment looking for signatures of dark matter scatterings off nuclei in its target $\mathrm{CaWO}_{4}$ crystals. The CRESST experiment operates detectors at $\mathrm{mK}$ temperatures. CRESST uses two signals from one particle interaction to detect particles: heat to measure the energy deposition of the incoming particle by the creation of phonons and scintillation light to identify the particle. We use a parameter called "light yield" to identify particles. Light yield is defined as the ratio between the en-

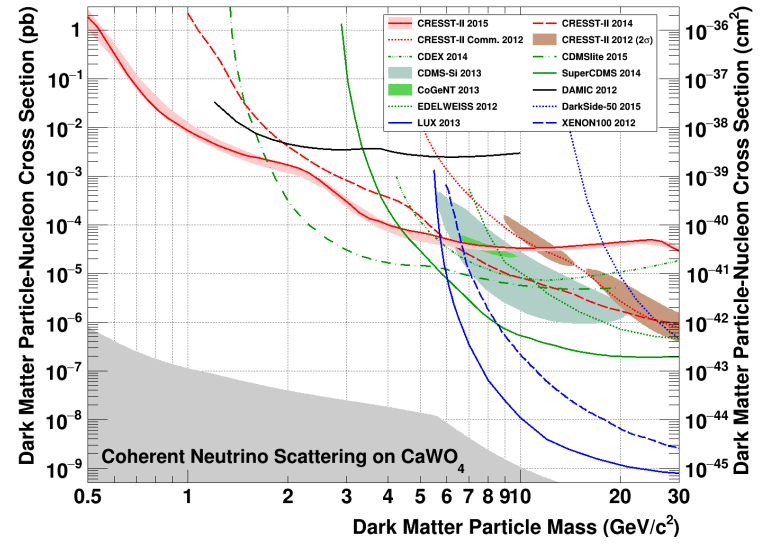

Figure 2: Spin-independent dark matter particlenucleon cross-section plot with respect to the dark matter particle mass [2].

\footnotetext{
* Speaker.

†Corresponding author: cenk.turkoglu@assoc.oeaw.ac.at

†Deceased
} 
ergy deposit in the light channel and the energy deposit in the phonon channel, normalised to 1 for gammas at $122 \mathrm{keV}$. An example plot of light yield drawn with respect to the recoil energy is given in Figure 3. In this figure, it is seen that there are different bands representing different event types. The grey band shows the electron recoils. Green and blue bands show recoils from Oxygen and Tungsten nuclei in $\mathrm{CaWO}_{4}$, respectively. The signal is expected to be a nuclear recoil. Therefore the region of interest (ROI) includes Oxygen, Calcium ${ }^{1}$ and Tungsten recoil bands. As indicated earlier, the CRESST experiment is searching for low mass dark matter particles [5] and for this, a range of interest of $0-40 \mathrm{keV}$ in recoil energy and light yield is the focus in dark matter analysis. However, as can be seen in Figure 3, the electron recoil band is overlapping with the nuclear recoil bands leading to a leakage of events in the ROI. In order to quantify the leakage, one has to know the background one is dealing with. For the background analysis, the data obtained with the TUM40 detector module [3, 4] are used.

\section{Radioactive Backgrounds in CRESST-II and Simulations}

Considering the setup of the experiment, one expects two different types of radioactive backgrounds: crystal internal backgrounds and external backgrounds. Internal backgrounds include natural radioactivity from alpha and beta/gamma decays as well as cosmogenically activated isotopes. External backgrounds include natural radioactivity in surrounding materials such as radioactivity coming from holders and the cryostat. In order to understand these backgrounds, an analysis was performed using Geant 4 modeling the backgrounds in the beta/gamma band [6]. In this analysis, beta/gamma emitters, such as ${ }^{210} \mathrm{~Pb}$ and ${ }^{227} \mathrm{Ac}$, have been found to contribute to the low end of the recoil energy spectrum. Cosmogenically activated ${ }^{179} \mathrm{Ta}$ has some contribution in

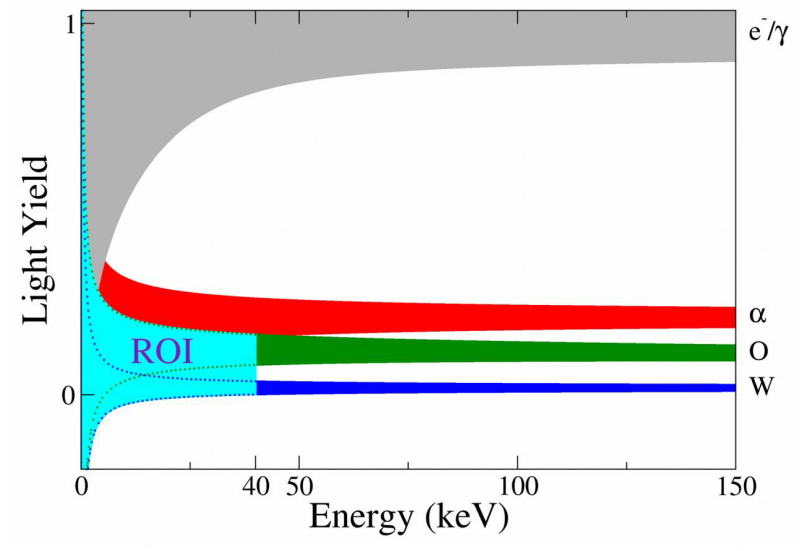

Figure 3: Light yield parameter drawn with respect to the recoil energy. The grey band shows the electron recoil band. Red band shows alpha interactions whereas recoils of Oxygen and Tungsten are shown in green and blue, respectively. The area marked as ROI is the region of interest for the dark matter particle search for the CRESST experiment. the form of peaks. Another obvious contribution comes from Copper fluorescence in the form of a line. The contribution of the ${ }^{210} \mathrm{~Pb}$ and ${ }^{227} \mathrm{Ac}$ isotopes to the Compton continuum is represented by the red dashed line in Figure 4. With all these contributions summed up, only $39 \%$ of the backgrounds can be explained. In order to have a more detailed understanding of the background, an analysis was performed on the alpha decays in the $\mathrm{MeV}$ energy regime. This analysis showed that there are contributions to the background from the ${ }^{238} \mathrm{U},{ }^{235} \mathrm{U}$ and ${ }^{232} \mathrm{Th}$ natural decay chains as can be seen on Figure 5. These chains include

\footnotetext{
${ }^{1}$ Calcium: Not drawn for clarity
} 
beta/gamma emitters which have contributions in the low energy region of CRESST as well as having high energy alpha lines. If one assumes radioactive equilibrium, the corresponding activities

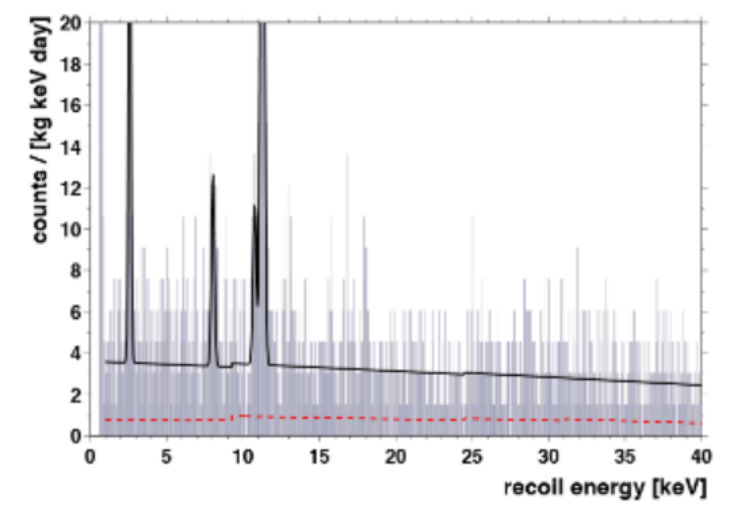

Figure 4: Radioactive backgrounds in TUM40 detector of CRESST-II Phase 2. Combination of ${ }^{210} \mathrm{~Pb}$ and ${ }^{227} \mathrm{Ac}$ decays are shown with red dashed line and the semi-empirical background model with black line [6].

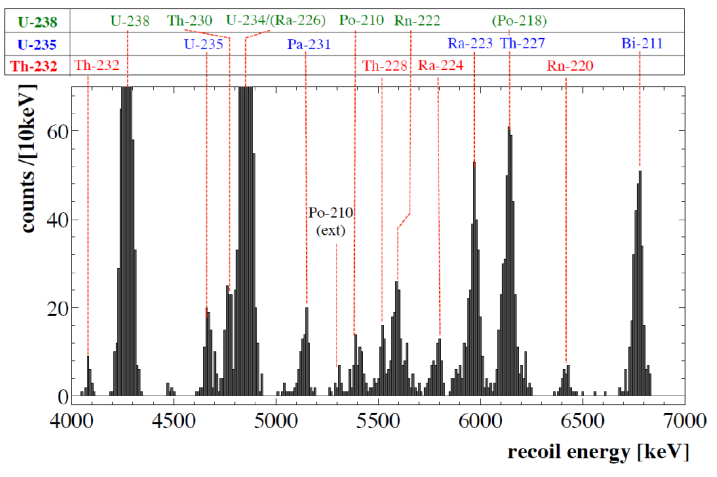

Figure 5: Alpha lines from the TUM40 data and the corresponding decay chains [6].

for beta/gamma emitters can be deduced from the activities of the alpha lines. For example, a ${ }^{238} \mathrm{U}$ isotope decays into ${ }^{234} \mathrm{Th}$ isotope which then decays into ${ }^{234} \mathrm{~Pa}$ via a beta decay. If the activity of ${ }^{238} \mathrm{U}$ or ${ }^{234} \mathrm{Th}$ is known, then the activity of the beta emitter ${ }^{234} \mathrm{~Pa}$ can also be determined. To understand the influence of these isotopes in the region of interest, simulations of both alpha emitters and beta/gamma emitters are performed. After implementing the TUM40 detector module in its full geometry in Geant4, each isotope is randomly distributed inside the $\mathrm{CaWO}_{4}$ crystal and the energy depositions of the decays are simulated. After performing fits to the alpha lines from the experimental data, one can derive the values of the alpha activities. The alpha lines from simulations are fit the same way and the activities measured in the experimental data can be used to normalize the simulation templates. Then, the activities of the beta/gamma emitters can be calculated. In addition to these internal contaminations, another background originates from the cosmogenically activated ${ }^{179} \mathrm{Ta}$ isotope. After proton capture, ${ }^{182} \mathrm{~W}$ decays into ${ }^{179} \mathrm{Ta}$ and this isotope decays into ${ }^{179} \mathrm{Hf}$ by electron capture. The existence of this isotope can be seen from sharp lines in the data. This isotope is simulated in the same way as the other internal background components. However, in this case, the activity will be calculated from the gamma lines of this isotope. In addition to the internal background contamination, external backgrounds should also be taken into account. While internal gamma events have accompanying beta spectra, external gamma lines can be identified from their sharp lines in data. When an isotope decays outside the crystal, the beta particles mostly cannot enter the crystal because of the $\mathrm{Cu}$ holders and the scintillating foil, but gamma rays can enter the crystal. This way, only a sharp line can be seen unlike an internally decaying isotope giving edges for gammas and a beta spectrum. A number of these lines were identified in an earlier analysis [6]. In order to simulate these lines, the isotopes in question are randomly distributed inside the copper holders of the detector module and the energy depositions of the decay products inside the $\mathrm{CaWO}_{4}$ crystal are recorded. After fitting the gamma ray peaks in the experimental data, the same fitting 
is done for the simulation and then simulation templates are normalized to the activities calculated from the experimental data. To compare the simulations with data, each individual simulation is summed up to give the total expected background.

\section{Latest Results from CRESST-II}

\subsection{Dark Matter Analysis}

In 2015, the CRESST collaboration released the latest dark matter results from CRESST-II Phase 2 [2]. These results were obtained from the data collected with the detector module named "Lise". The most important feature of this detector module is that it had the lowest recoil energy threshold of all the detectors operated in Phase 2. With a recoil energy threshold of $307 \pm 4$ $\mathrm{eV}$, the Lise detector module reached a sensitivity for low mass dark matter particles no other direct detection experiment had been able to do. $52 \mathrm{~kg} *$ days of gross exposure was used for the dark matter analysis and the background level in the energy range between $1-40 \mathrm{keV}$ was 8.5 counts/(kg*keV*day). With this analysis, CRESST was able to probe dark matter particle masses as low as $500 \mathrm{MeV} / \mathrm{c}^{2}$. The upper limit achieved with this analysis can be seen in Figure 2.

\subsection{Dark Photon Analysis}

One of the theories claims that dark matter could couple to hypothetical long-lived vector particles called dark photons [7, 8]. U(1) extension of the standard model introduces dark photons as dark matter candidates. However, since these particles have different properties, a different type of analysis was performed compared to a WIMP-like dark matter particle analysis. While a dark matter particle scatters off nuclei and only a part of its energy is absorbed, with dark photons, their total energy is absorbed. In addition, since a WIMP-like dark matter particle

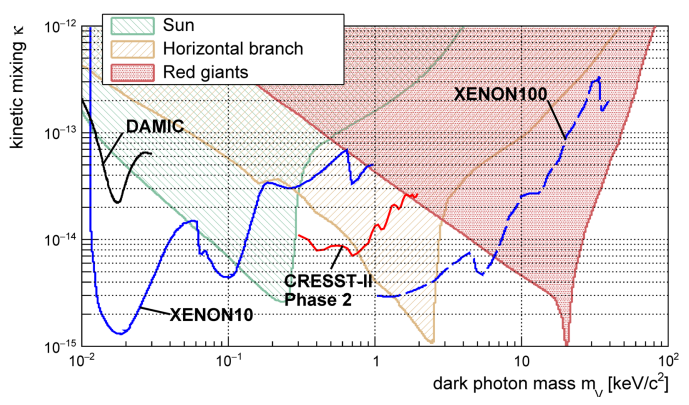

Figure 6: Upper limits on the kinetic mixing of dark photons [9]. scatters off nuclei, the signal is expected to be in the nuclear recoil band whereas dark photon-like dark matter is absorbed by electrons and the possible signal should be found at its rest mass in the electron recoil band. A peak search was done using the Lise detector data with a $52 \mathrm{~kg} *$ days exposure before cuts [9]. The resulting upper limit can be seen in Figure 6. As seen in the figure, a new parameter space between dark photon masses in the range of $300-1000 \mathrm{eV}$ was probed and a new upper limit was established in this region [9].

\section{Current Status of the CRESST Experiment}

Based on the experience acquired with CRESST-II Phase 2, new types of detectors were constructed. These new detector modules have two important features. The mass of the $\mathrm{CaWO}_{4}$ crystal was reduced from 250-300 grams to 25 grams in order to achieve a lower recoil energy threshold. 
The design goal for the energy threshold was $100 \mathrm{eV}$. The second important feature concerns a fully active veto [10]. CRESST-III Phase 1 has been taking physics data since November 2016. After checking the first data of this phase, it was understood that the $100 \mathrm{eV}$ recoil energy threshold goal has been exceeded.

\section{Conlusion}

Dark matter is one of the biggest unknowns in our universe and the CRESST experiment has been looking for signals from dark matter candidates in the low mass range. In this area, the latest results of CRESST-II for low mass dark matter particles are world leading. However, knowing the types and the amounts of backgrounds is crucial for a rare event search. To better understand the backgrounds, the development of a comprehensive background model based on GEANT4 simulations is significant. With the data from CRESST-II detector Lise, three different analyses were performed in the past two years: a dark matter analysis in the standard framework [2], a momentum-dependent dark matter analysis [11] and a dark photon analysis [9], all of which have been world leading in terms of limits on the low mass dark matter candidates. In 2016, a new batch of detector modules was installed and CRESST-III Phase 1 started taking data. So far, the energy threshold goal of $100 \mathrm{eV}$ has been exceeded and some new results are already available [12].

\section{References}

[1] H. Baer, K. Y. Choi, J. E. Kim and L. Roszkowski, "Dark matter production in the early Universe: beyond the thermal WIMP paradigm", Phys. Rept. 555 (2015) 1 doi:10.1016/j.physrep.2014.10.002 [arXiv:1407.0017 [hep-ph]].

[2] G. Angloher et al. [CRESST Collaboration], "Results on light dark matter particles with a low-threshold CRESST-II detector", Eur. Phys. J. C 76 (2016) no.1, 25 doi:10.1140/epjc/s10052-016-3877-3 [arXiv:1509.01515 [astro-ph.CO]].

[3] R. Strauss et al. [CRESST Collaboration], "A detector module with highly efficient surface-alpha event rejection operated in CRESST-II Phase 2", Eur. Phys. J. C 75 (2015) no.8, 352 doi:10.1140/epjc/s10052-015-3572-9 [arXiv:1410.1753 [physics.ins-det]].

[4] G. Angloher et al. [CRESST-II Collaboration], "Results on low mass WIMPs using an upgraded CRESST-II detector”, Eur. Phys. J. C 74 (2014) no.12, 3184 doi:10.1140/epjc/s10052-014-3184-9 [arXiv:1407.3146 [astro-ph.CO]].

[5] G. Angloher et al. [CRESST Collaboration], "Probing low WIMP masses with the next generation of CRESST detector", arXiv:1503.08065 [astro-ph.IM].

[6] R. Strauss et al. [CRESST Collaboration], "Beta/gamma and alpha backgrounds in CRESST-II Phase 2”, JCAP 1506 (2015) no.06, 030 doi:10.1088/1475-7516/2015/06/030 [arXiv:1410.4188 [physics.ins-det]].

[7] An H. et al., "Direct Detection Constraints on Dark Photon Dark Matter", Physics Letters B, Volume 747, Pages 331-338, 30 July 2015.

[8] Pospelov M. et al., "Bosonic super-WIMPs as keV-scale dark matter", Phys. Rev. D 78, 115012, 16 December 2008. 
[9] G. Angloher et al. [CRESST Collaboration], "Dark-Photon Search using Data from CRESST-II Phase 2", Eur. Phys. J. C 77 (2017) no.5, 299 doi:10.1140/epjc/s10052-017-4878-6 [arXiv:1612.07662 [hep-ex]].

[10] R. Strauss et al., "The CRESST-III low-mass WIMP detector”, J. Phys. Conf. Ser. 718 (2016) no.4, 042048. doi:10.1088/1742-6596/718/4/042048

[11] G. Angloher et al., "Limits on momentum-dependent asymmetric dark matter with CRESST-II", Phys. Rev. Lett. 117 (2016) no.2, 021303 doi:10.1103/PhysRevLett.117.021303 [arXiv:1601.04447 [astro-ph.CO]].

[12] Petricca F. et al., "Direct dark matter search with the CRESST-III experiment", TAUP 2017 XV. International Conference on Topics in Astroparticle and Underground Physics, 24-28 July 2017, proceedings to be published. 